\title{
Intraoperative $\beta^{-}$Detecting Probe For Radio-Guided Surgery in Tumour Resection
}

\author{
Elena Solfaroli Camillocci*†, Fabio Bellini*†, Valerio Bocci ${ }^{\dagger}$, Francesco Collamati ${ }^{\dagger \dagger}$, Erika De Lucia ${ }^{\S}$, \\ Riccardo Faccini*†, Michela Marafini ${ }^{\dagger \uparrow}$, Ilaria Mattei ${ }^{\S} \|$, Riccardo Paramatti ${ }^{\dagger}$, Vincenzo Patera ${ }^{\dagger \ddagger}$, Davide Pinci ${ }^{\dagger}$, \\ Luigi Recchia ${ }^{\dagger}$, Aandrea Russomando* ${ }^{* * *}$, Alessio Sarti ${ }^{\ddagger \S}$, Adalberto Sciubba ${ }^{\dagger \ddagger}$, Martina Senzacqua*†, \\ Cecilia Voena $^{\dagger}$ and Silvio Morganti ${ }^{\dagger}$
}

\begin{abstract}
The development of the $\beta^{-}$based radio-guided surgery aims to extend the technique to those tumours where surgery is the only possible treatment and the assessment of the resection would most profit from the low background around the lesion, as for brain tumours. Feasibility studies on meningioma and gliomas already estimated the potentiality of this new treatment. To validate the technique, a prototype of the intraoperative probe detecting $\beta^{-}$decays and specific phantoms simulating tumour remnant patterns embedded in healthy tissue have been realized. The response of the probe in this simulated environment is tested with dedicated procedures. This document discusses the innovative aspects of the method, the status of the developed intraoperative $\beta^{-}$detecting probe and the results of the preclinical tests.
\end{abstract}

\section{INTRODUCTION}

$\mathbf{T}$ HE main advantage of the radio-guided surgery (RGS) technique exploiting $\beta^{-}$radiation [1], [2], compared to the traditional RGS using $\gamma$ radiation [3], is a more favourable ratio between the signal coming from the tumor and the rest of the body. Conversely to the tracers with $\gamma$ emitters, pure $\beta^{-}$radionuclides emit electrons which penetrate only a few millimeters of tissue and produce scarce gamma radiation (the bremsstrahlung contribution above $100 \mathrm{keV}$ being less than $0.1 \%$ ) resulting in a very low background on the lesion signal.

Operating in a low background environment allows the development of a handy and compact probe which, detecting particles emitted locally, provides a clearer delineation of margins of the lesioned tissue. The RGS technique applicability can therefore be extended also to cases with large uptake of the tracer from healthy organs next to the lesion, minimizing the radiotracer activity to be administered with obvious extremely positive implications for the patients. Low exposure of the medical team is also expected [1].

Brain tumors are one of the clinical application that would most profit from the technique with $\beta^{-}$radiation since a complete removal of the tumor is critical to reduce relapse and other RGS techniques are limited by the significant uptake of the healthy brain. Meningiomas have been identified as the first clinical case of interest for the technique validation because

* Dip. Fisica, Sapienza Univ. di Roma, Rome, Italy; ${ }^{\dagger}$ INFN Sezione di Roma, Rome, Italy; $\ddagger$ Dip. Scienze di Base e Applicate per l'Ingegneria, Sapienza Univ. di Roma, Rome, Italy; $\S$ Laboratori Nazionali di Frascati dellINFN, Frascati, Italy; M Museo Storico della Fisica e Centro Studi e Ricerche "E. Fermi", Rome, Italy; " Dipartimento di Fisica, Università Roma Tre, Rome, Italy; ** Center for Life Nano Science@Sapienza, Istituto Italiano di Tecnologia, Rome, Italy. of their sensitivity to DOTATOC [4], a synthetic analogue of somatostatin, that can be labelled with ${ }^{90} \mathrm{Y}$, a pure $\beta^{-}$ radionuclide [5]. The ${ }^{90} \mathrm{Y}$ half-life $(64 \mathrm{~h})$ and electron energy spectrum (endpoint: $2.28 \mathrm{MeV}$ ) make this isotope suitable for nuclear medicine applications and, in particular, for $\beta^{-}$RGS due to absence of $\gamma$ emission. A study [6] of the potentiality of the proposed RGS technique was performed using PET diagnostic exams of patients affected by meningioma after administration of ${ }^{68} \mathrm{Ga}$-DOTATOC (the uptake can be assumed independent from the linked radionuclide). The study of the uptake of the radiotracer nearby the lesion showed that a tumor-non-tumor ratio (TNR) greater than 10 is observed in almost all the cases and a detailed simulation of the intraoperative probe allowed predictions of the RGS performance that resulted as very promising. Similar conclusions were recently achieved on neuro-endocrine tumors [7].

To implement and validate this innovative RGS technique, we have developed and characterized an intraoperative probe detecting $\beta^{-}$decays. Moreover, we have realized specific phantoms simulating tumour remnant pattern embedded in healthy tissue and developed dedicated test procedures to investigate the response of the probe in this simulated environment. This article describes the developed intraoperative $\beta^{-}$detecting probe and its performance and the results of the preclinical tests.

\section{THE $\beta^{-}$INTRAOPERATIVE PROBE}

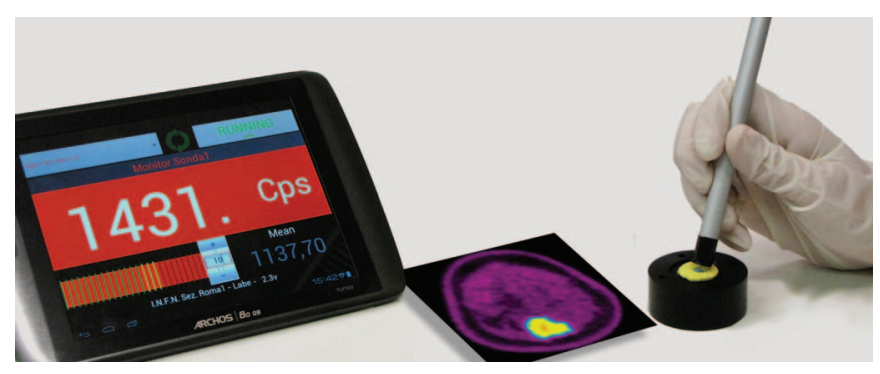

Fig. 1. Image of the intraoperative $\beta^{-}$probe.

The first prototype of the intraoperative probe detecting $\beta^{-}$decays (Fig. 1) was designed a compact device with high sensitivity to low energy electrons, directional detection and fast response (few seconds) compatible with the surgical activity. The required target spatial resolution is fixed by the typical volume $(0.1 \mathrm{ml})$ of a surgical tumour residual. 
The radiation sensitive element of the $\beta^{-}$probe is a small scintillator tip made of commercial poly-crystalline para-terphenyl doped to $0.1 \%$ in mass with diphenylbutadiene [8] manufactured by Detec-Europe. This material was adopted, after a detailed study [9], due to its high light yield ( $\sim 3$ times larger than typical organic scintillators), nonhygroscopic property, and low density that minimizes the sensitivity to photons. Different detector sizes were tested: the best configuration resulted in a cylinder of $5 \mathrm{~mm}$ in diameter and $3 \mathrm{~mm}$ in height.

The scintillator tip is shielded from radiation coming from the sides by a black PVC ring with external diameter of $11 \mathrm{~mm}$. A $10 \mu \mathrm{m}$-thick aluminium front-end sheet covers the detector window to ensure light tightness. This assembly is mounted on top of an easy-to-handle aluminum cylindrical body (diameter $8 \mathrm{~mm}$ and length $14 \mathrm{~cm}$ ).

In order to avoid the risk of patients coming into contact with electrical devices, the scintillation light is guided by four $50 \mathrm{~cm}$ long optical fibres outside the probe to a Hamamatsu H10721-210 photo-multiplier tube (PMT). This photo-sensor module has an integrated high voltage power supply circuit requiring an input voltage as low as $5 \mathrm{~V}$, making this device compatible with the surgical environment. Finally, the readout electronics and the logic board are housed in a compact box that wirelessly connects to a remote monitor to display the counting rate.

\section{Characterization of the $\beta^{-}$intraoperative PROBE}

The detecting performances of the developed intraoperative probe are studied in laboratory using different source topologies and emitting radionuclides.

\section{A. Sensitivity to $\beta^{-}$decays}

The probe sensitivity in detecting electrons from $\beta^{-}$decays is measured with a point-like ${ }^{90} \mathrm{Sr}$ source with nominal activity of $370 \mathrm{~Bq}$ (Sr-source). The ${ }^{90} \mathrm{Sr}$ spectrum is made of two components in secular equilibrium: the $\mathrm{Sr} \beta^{-}$decay to ${ }^{90} \mathrm{Y}$ and the subsequent $\beta^{-}$decay of the daughter. The latter component is exactly the one proposed for this application. The rate measured by the probe is $3.8 \cdot 10^{5} \mathrm{cps} / \mathrm{MBq}$ resulting in a detection efficiency of $40 \%$ of the $\beta^{-}$decay products of the decay chain ${ }^{90} \mathrm{Sr} /{ }^{90} \mathrm{Y}$.

A relevant parameter for the definition of the probe sensitivity is the detectable $\beta^{-}$energy threshold that is the minimal kinetic energy of the electron that reaches the probe and is detected. To evaluate this threshold, a vertical scan over the Sr-source has been performed measuring the rate with both the source and the probe in water for several distances between the two ("scan in water"). The presence of increasing amounts of water dumps the $\beta^{-}$energy spectrum in a known way and the minimum amount of water for which no signal is observed allows the inference of the detection threshold. Fig. 2 shows the measured rate as a function of the probe distance from the source at high distances: the probe counting rate is compatible with the dark noise rate at $8.5 \mathrm{~mm}$ over the source.

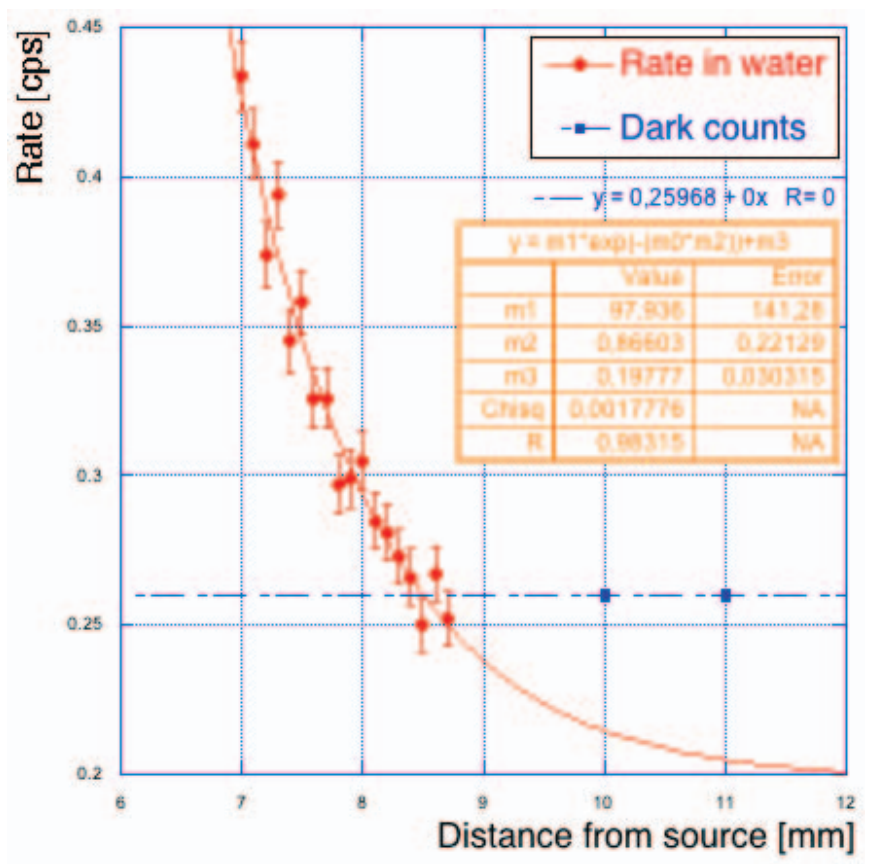

Fig. 2. Large distance tail of the vertical scan over the Sr-source in water. The blue horizontal line represents the dark counting rate.

To evaluate the electron energy loss along this path and the residual energy of the electron approaching to the probe, a Monte Carlo simulation based on FLUKA code [10] is used. The FLUKA software fully simulates the energy spectrum emitted by the point-like Sr source, the energy deposition in water and the probe. Then, the energy spectrum of electrons reaching the detector volume is evaluated at different distances from the source and the "theoretical" rate is evaluated by calculating the integral of the electrons having an energy greater than a certain threshold, varying it from 300 to $600 \mathrm{keV}$ (consistent with what expected for a $2 \mathrm{MeV}$ electron crossing $8 \mathrm{~mm}$ thickness of water). To estimate the energy threshold which best reproduces the data, a $\chi^{2}$ test on point by point basis is performed and by means of its minimization the value of $487 \pm 33 \mathrm{keV}$ for the energy threshold is obtained. That allows us to conclude that the probe shows a high sensitivity for electrons with energy above $500 \mathrm{keV}$ and it is, therefore, largely efficient ( $>80 \%$ according to the efficiency mentioned above) in the energy range of the electrons emitted by ${ }^{90} \mathrm{Y}$, which is the radionuclide that would be exploited for the RGS.

\section{B. Spatial resolution}

To study the capability of the probe to reconstruct active spots, the detector is mounted on a motorized linear actuator ensuring position accuracy of $1.5 \mu \mathrm{m}$ and horizontal scans are performed over point-like and extended sources.

The scan over the point-like Sr-source (Fig. 3) shows how spatial resolution depends on the distance between the probe tip and the surface. The reconstructed profiles measured at distances ranging from $350 \mu \mathrm{m}$ to $1.8 \mathrm{~mm}$ are fitted using a Gaussian distribution obtaining $\sigma$ between 2.8 and $4.4 \mathrm{~mm}$.

The scan over the extended source (Fig. 4), instead, explores the discovery power of the probe in a more realistic simulation 


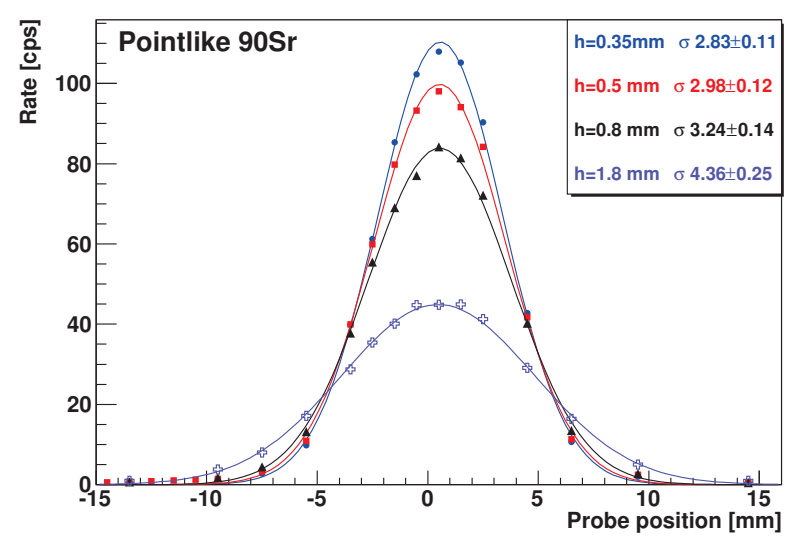

Fig. 3. Profiles of the point-like Sr-source reconstructed by the probe at different distances from the source.

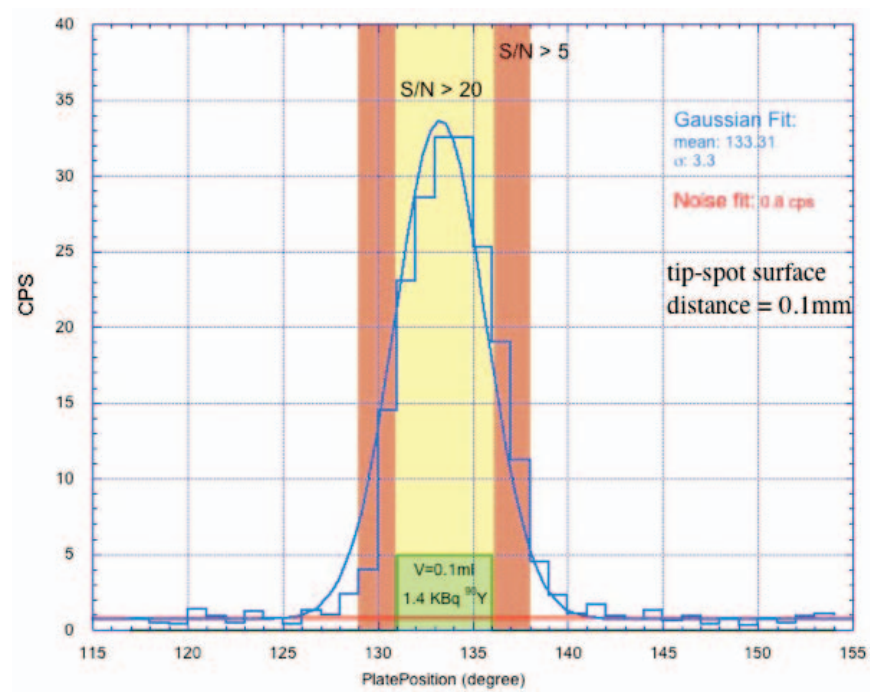

Fig. 4. Lateral scan of a $1.4 \mathrm{kBq}, 0.1 \mathrm{ml},{ }^{90} \mathrm{Y}$ phantom. Gaussian and linear fits are for the signal and dark count rate respectively. See text for the band definition.

of a radio-labelled tumour residual. A cylindrical phantom with volume of $0.1 \mathrm{ml}$ (diameter: $6.0 \mathrm{~mm}$, height: $3.5 \mathrm{~mm}$ ) is filled with a saline solution with ${ }^{90} \mathrm{Y}$ radionuclide of $1.4 \mathrm{kBq}$ activity, comparable to those administered for diagnostic purposes. The profile reconstructed by the probe is obtained with an horizontal scan of $1.5 \mathrm{~mm}$ steps and $10 \mathrm{~s}$ per position and a distance between the probe and the phantom surface of $100 \mu \mathrm{m}$. The probe is able to correctly identify the active spot with a signal discriminating threshold $\mathrm{S} / \mathrm{N}>5$, where $\mathrm{S}$ is the number of detected events and $\mathrm{N}$ is the number of dark counts, and the real phantom dimension is reconstructed with $\mathrm{S} / \mathrm{N}>20$.

\section{Background rejection capability}

The usage of pure $\beta^{-}$emitting tracers allows the operation with low radiation background, the residual being mainly due to photons coming from the Bremsstrahlung radiation of the electrons penetrating the tissue. The abundance of the expected Bremsstrahlung radiation in the tissue as a function
Figure 1 from Xing Rong et al 2012 Phys. Med. Biol. 573711

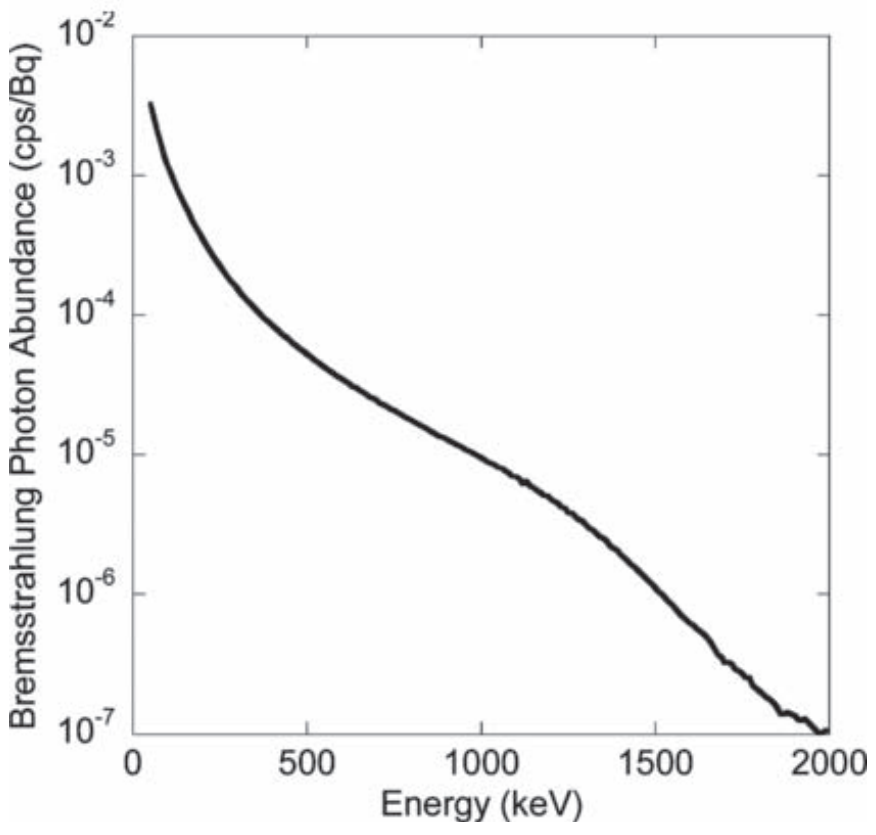

Fig. 5. Energy spectrum (50-2000 keV, $10 \mathrm{keV}$ interval) of ${ }^{90} \mathrm{Y}$ Bremsstrahlung photons in water.

of the photon energy is shown in Fig. 5 as computed with a simulation of a ${ }^{90} \mathrm{Y}$ source in water [11].

The sensitivity of the probe to these photons is measured using three point-like sources: ${ }^{133} \mathrm{Ba}$ emitting photons with energy ranging from 80 to $350 \mathrm{keV},{ }^{137} \mathrm{Cs}$ with gamma emission at $\mathrm{E}_{\gamma}=662 \mathrm{keV}$ and ${ }^{60} \mathrm{Co}$ with $\mathrm{E}_{\gamma 1}=1170 \mathrm{keV}$ and $\mathrm{E}_{\gamma 2}=1330 \mathrm{keV}$. To avoid signal from electrons in the case of Cs decays, three copper layers with $350 \mu \mathrm{m}$ thickness are inserted in sequence between the source and the probe tip, and the measurements are repeated at each step. The counts as measured by the probe are shown in the Fig. 6 for the three sources. Except for the first measure on the Cs source, introducing the copper absorbers implies a very small decrease in rate compatible with the attenuation in copper and the change in geometrical acceptance.

The sensitivity to the photons emitted by the ${ }^{133} \mathrm{Ba}$ source is below $10^{-5}$, whereas for the $\gamma \mathrm{s}$ from the ${ }^{137} \mathrm{Cs}$ and ${ }^{60} \mathrm{Co}$ sources the sensitivity is still below $10^{-4}$. These measurements allow us to conclude that the intraoperative $\beta^{-}$probe is not sensitive to the Bremsstrahlung photons and therefore the effectiveness of the RGS technique would not be affected by this background.

\section{PRECLINICAL TESTS ON SPECIFIC PHANTOMS}

To estimate the performances of the technique, the probe is tested on specific phantoms reproducing finite size meningioma remnants embedded in healthy brain tissue with a TNR as expected in a real clinical case. The same set-up allows going through different kinds of interfaces and visual/acoustic feedback to identify which one could best assist the surgeon in the search for infected residuals. 


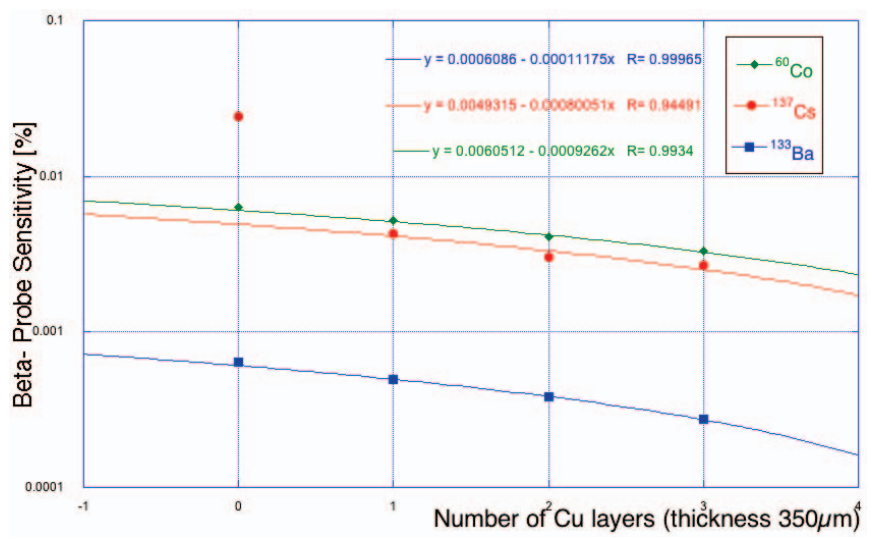

Fig. 6. Probe sensitivity to photons emitted by three different sources: ${ }^{60} \mathrm{Co}$ $\mathrm{E}_{\gamma 1}=1170 \mathrm{keV} \mathrm{E} \mathrm{E}_{\gamma 2}=1330 \mathrm{keV},{ }^{137} \mathrm{Cs}_{\gamma}=662 \mathrm{keV},{ }^{133} \mathrm{Ba} \mathrm{E}_{\gamma}$ ranging from 80 to $350 \mathrm{keV}$. The measurements are repeated after insertions of copper layers with thickness of $350 \mu \mathrm{m}$ between the source and the probe tip to absorb the electronic component of the Caesium emissions.

\section{A. Realization of the phantoms}

The surgical environment is simulated saturating conveniently shaped cuttings of a commercially available sponge (Wettex Classic by Vileda ${ }^{\circledR}$ ) with leftover of the ${ }^{90} \mathrm{Y}$ DOTATOC administered to patients during normal clinical activity. The liquid source is diluted to reach the mass and concentration $(10 \mathrm{kBq} / \mathrm{ml})$ allowed by law for the transport and usage of this $\beta$ emitter without special constraints.

The sponge is made of $65 \%$ cellulose and $35 \%$ cotton fibres packed as $20 \times 20 \mathrm{~cm}$ wide and $2 \mathrm{~mm}$ thick dry sheets. The relevant parameters driving our choice were:

- water absorbency (as measured by us on small samples);

- ease of obtaining a sharp and precise cut even with few millimetre wide complex shapes;

- capability of a sample to regain its original dimensions after a wetting-drying cycle.

To radio-protect operators, the phantoms are set up and used within a $10 \mathrm{~mm}$ thick PMMA glove box.

With this technique, both wet or dry surfaces can be produced depending on the scope of the test:

- dry phantoms are used to test the neat physics detector performances (e.g. sensitivity to active spots, capability of profile reconstruction);

- wet surfaces are suitable for studying and optimizing the probe usage in a more realistic environment where, for example, tip contamination might occur adding a random background to the signal coming from the tissues.

The simplest assembly made with this technique is shown in Fig. 7: a $5 \mathrm{~mm}$ diameter circular cutting simulating a $0.05 \mathrm{ml}$ tumour residual is inserted inside a larger $(20 \mathrm{~mm}$ diameter) ring reproducing the nearby healthy tissue. A third round shape (disk) is eventually used to reproduce the presence of further healthy tissues above or under the hot spot. Different TNR between the tumour and the surrounding healthy tissue are realised drenching the cuttings, up to saturation, with the properly diluted ${ }^{90} \mathrm{Y}$ solution. The elements are then dried and combined to shape the topology of interest (Fig. 8).

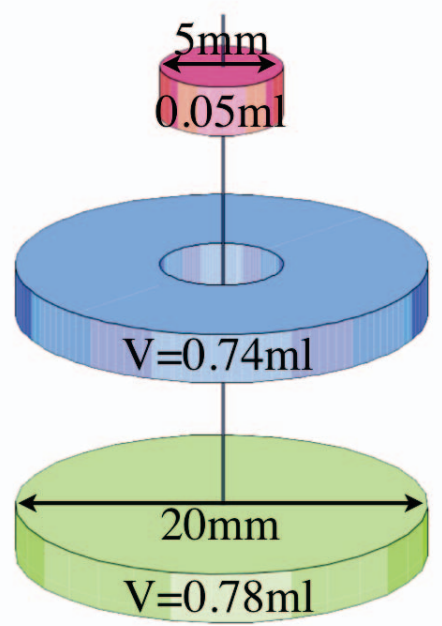

Fig. 7. Assembling of a phantom simulating a tumour residual of $0.05 \mathrm{ml}$ embedded in surrounding (the ring) and underneath (the disk) healthy tissue.

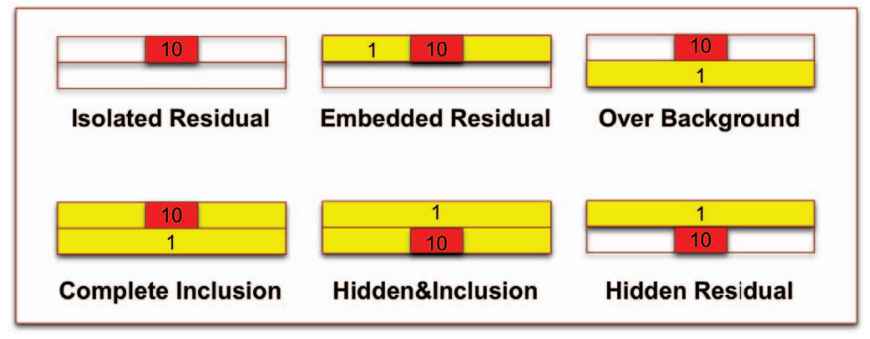

Fig. 8. Varying position and activity (white: non-active tissue, yellow: active tissue) of the assembly elements the discovery potential of the probe can be tested on surfaces reproducing, even if schematically, some possible combinations of cancerous residual location and TNR occurring during surgery.

The nominal activity of any phantom is inferred from the activity of the ${ }^{90}$ Y-DOTATOC vial at the time of delivery and the dilution factor of the bath prepared to drench the samples. The relative activity of the final dried phantoms is individually measured before forming the assembly.

\section{B. Active spot identification}

A scan over the described assembly is performed fixing the probe to a XZ motorised system. The X linear actuator runs the probe over the phantom in $1 \mathrm{~mm}$ steps, with a measurement time of $10 \mathrm{~s}$ per position, while the $\mathrm{Z}$ actuator is used to set the tip to surface distance with a precision of $1.5 \mu \mathrm{m}$.

The different configurations are analysed in terms of falsepositive probability, i.e. the probability that the signal (cps) emitted by an healthy tissue is incorrectly flagged by the probe as tumour residual. In the actual case, this flag will be set in real time by the system comparing the signal measured over the tissue under investigation and the patient reference background previously measured over their healthy tissue. The probability of false-positive then depends on the probe efficiency, the time to response and the actual TNR.

An example of a scan with an indication of the false-positive probability is shown in Fig. 9. The horizontal lines indicate 
the threshold (in cps) level over which at 90\%, 95\% and 99\% confidence level (C.L.) the underlying area is flagged as "tumour" with a measure lasting $1 \mathrm{~s}$ or $10 \mathrm{~s}$.

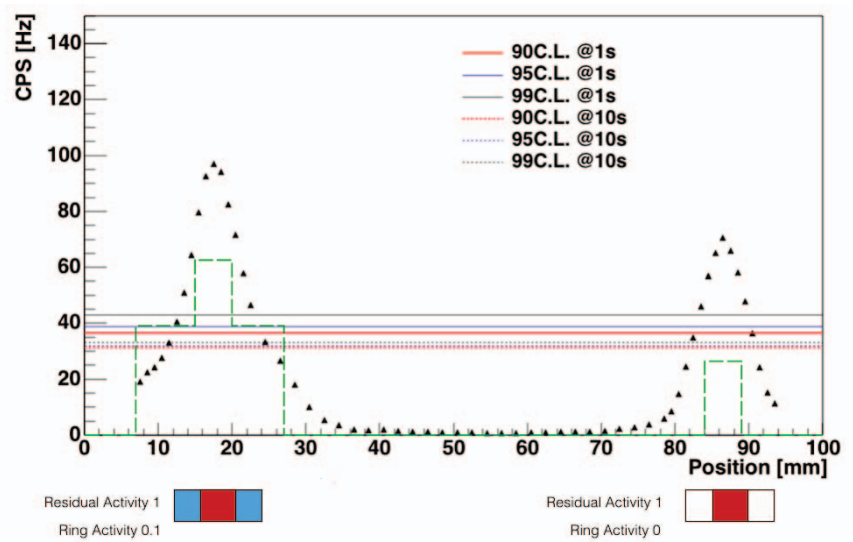

Fig. 9. Scan over two assemblies with a probe tip-surface distance next to zero. The left phantom simulates a $0.5 \mathrm{ml}$ tumour surrounded by a same thickness healthy tissue with a TNR of 10 (as observed in patients affected by meningioma), the second assembly is an isolated residual (non-active surrounding tissue).

Fig. 10 and 11 show the same measurement performed on different tumour-health tissue patterns (fixed the specific activity and the TNR) that might affect the discovery potential of the probe.

These evaluations are performed in a neat and well known configuration, whereas the surgical environment might introduce some perturbations as, for example, a contamination of the probe tip coming into contact with the wet active tissue. Such effect can be evaluated directly from the scan shown in Fig. 9 adding, via software, an extra background to the measure up to the minimum TNR that makes still detectable the hot spot. The study shows that the probe is able to correctly identify the active spot also in case of TNR as low as 2 with a $99 \%$ C.L. and a time of measure of $10 \mathrm{~s}$. That means that an eventual contamination would not be a problem if monitored with frequent background calibration during the operation.

Moreover, these results imply that the RGS technique with $\beta^{-}$decays would be effective also in case of patients with a lower TNR compared to those affected by meningioma, and therefore they are a confirm that the RGS could be extended to clinical case of interest with an eventual uptake of the radiotracer in the nearby healthy organs [6].

\section{Minimum detectable tumour residual}

A different phantom pattern is used to simulate and study the evolution of the probe response while removing an extended tumour area aiming at the identification of the minimum detectable residual. For this purpose the assembly shown in Fig. 12 is used. A scan over the whole strip is repeated reducing the tumoural area by removing a tile at once starting from the wider one $(5 \mathrm{~mm})$. At each iteration the assembly is packed again to always keep the tumour area enclosed between healthy tissues.

The scan over the full $15 \mathrm{~mm}$ long tumour phantom is shown in Fig. 13. Following the scan from the right to the

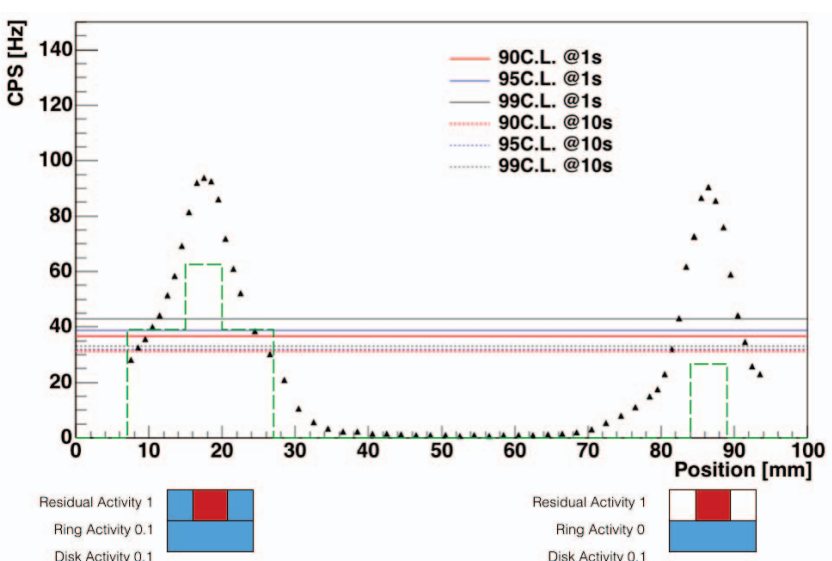

Fig. 10. Scan over two assemblies with a probe tip-surface distance close to zero realized to study the sensitivity of the probe to a $0.5 \mathrm{ml}$ hot spot masked by side and beneath emitting $(\mathrm{TNR}=10)$ healthy tissue.

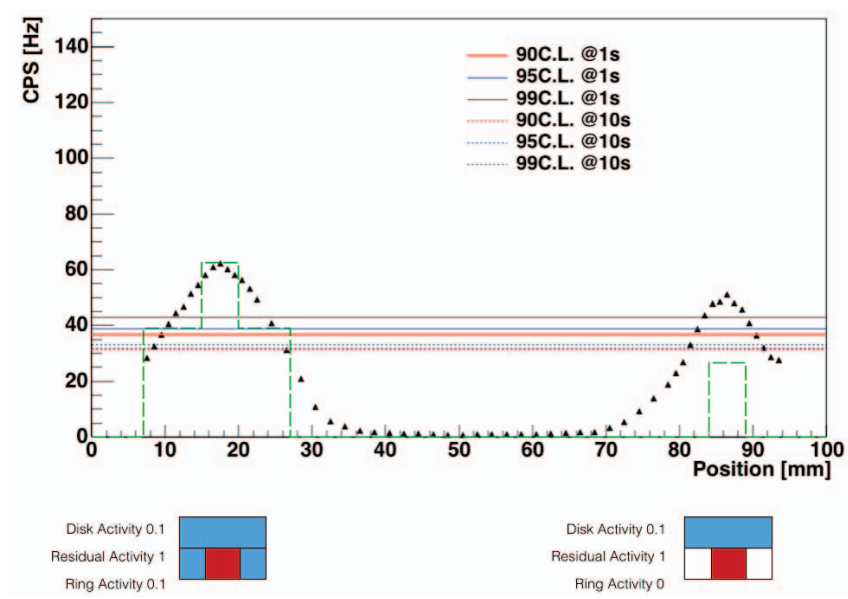

Fig. 11. Scan over two assemblies with a probe tip-surface distance equal to zero to test the sensitivity of the probe to a hot spot hidden under an emitting $(\mathrm{TNR}=10)$ healthy tissue.

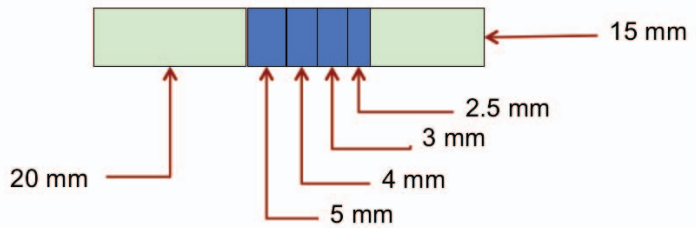

Fig. 12. The phantom prepared to simulate the progress of a surgical removal of a tumour is made by a strip (blue) of seven tiles of different sizes and charged at the tumour uptake. A sequence of scans is performed removing a strip at each iteration to study the minimum detectable residual. At the beginning and the end of this assembly two tiles (green) ten times less active define the boundary of the healthy tissue surrounding the tumour. 
left the experimental shape is read as follow: the cps counts start from zero (the probe is outside the scanning area) and increase as the probe goes over the healthy tissue. The small plateau at $30 \mathrm{cps}$ defines the background level of this measure. Approaching the tumour phantom, the counts increase up to plateau at about $250 \mathrm{cps}$ lasting as much as the probe sensitive area is fully illuminated by the tumour area. As soon as the sensitive detector exits the last tumour tile the rate goes back to that of the healthy tissue.

The response measured reducing the tumour thickness to $3 \mathrm{~mm}$ and $2.5 \mathrm{~mm}$ are shown in Fig. 14 and Fig. 15 respectively. In both cases the tumour margins are clearly identified once the activity of the surrounding healthy tissue is known.

The smallest residual tested in this measure, obtained reducing by $5 \mathrm{~mm}$ the length of the $2.5 \mathrm{~mm}$ tile, has a volume of $0.03 \mathrm{ml}$. The corresponding signal is shown in Fig. 15 .

\section{CONCLUSION}

We report on the performance of the first prototype of the intraoperative probe purposely designed to exploit the newly proposed radio-guided surgery with $\beta^{-}$decays. The low density and the high light yield of the para-terphenyl scintillator allow the development of a very compact device with a good sensitivity to electrons in the range of the ${ }^{90} \mathrm{Y}$ decays and almost transparent to the bremsstrahlung photons. The first results obtained experimenting the probe on pointlike and extended ${ }^{90} \mathrm{Y}$ sources highlight the potentiality of this approach in all the issues relevant in this field: millimetric remnants discovery potential, spatial resolution, fast response, dose administered to the patient, operators safety.

\section{REFERENCES}

[1] Solfaroli Camillocci E et al 2014 Sci. $\quad$ Rep. 444401 DOI: $10.1038 /$ srep04401

[2] Patent PCT/IT2014/000025 deposited by Università degli studi di Roma "La Sapienza", Istituto Nazionale di Fisica Nucleare and Museo storico della fisica e centro studi e ricerche "E. Fermi".

[3] Povoski SP et al 2009 World Journal of Surgical Oncology 7-11

[4] Bartolomei M et al 2009 Eur J Nucl Med Mol Imaging 36 1407-16

[5] Heppeler A et al 1999 Chem Eur J 7 1974-81

[6] Collamati F et al 2015 J Nucl Med 56 1-6 DOI:10.2967/jnumed.114.145995

[7] Collamati F et al 2015 "Time evolution of DOTATOC uptake in Neuroendocrine Tumors in view of a possible application of Radio-guided Surgery with $\beta^{-}$Decays." Submitted to J Nucl Med

[8] Budakovsky SV, Galunov NZ, Grinyov BV, Kim JK, Kim YK, Tarasenko OA 2009 Functional Materials 16, 1 86-91

[9] Angelone M et al 2014 IEEE Transactions on Nuclear Science 61, 3 1483-87 DOI:10.1109/TNS.2014.2322106

[10] Ferrari A, Sala PR, Fasso A, Ranft J 2005 Tech. Rep. CERN-2005-10, INFN/TC05/11, SLAC-R-773

[11] Rong X, Du Y, Frey EC 2012 Phys Med Biol 57371125 DOI:10.1088/0031-9155/57/12/3711

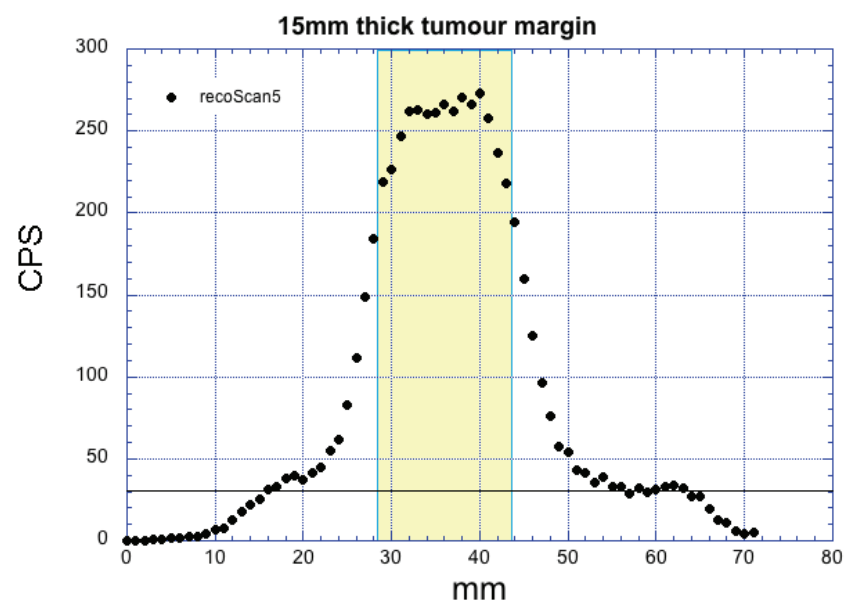

Fig. 13. A scan over a $15 \times 10 \mathrm{~mm}^{2}$ tumour activated phantom. The yellow area indicate the position of the tiles between the two healthy tissue phantoms. The limited lateral shield makes the probe sensitive to the approaching active area about $4 \mathrm{~mm}$ before the detector goes over the hot tiles.

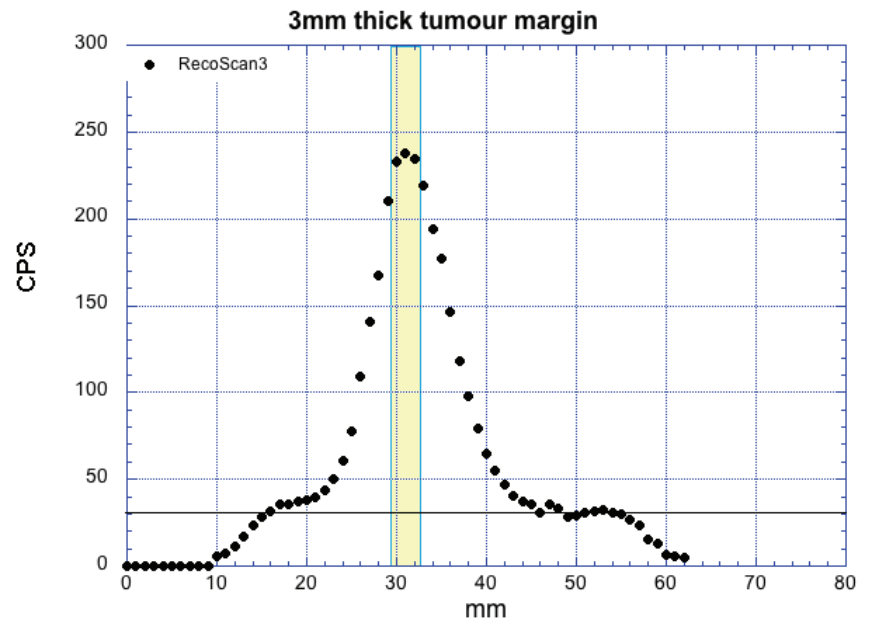

Fig. 14. Scan over a $3 \mathrm{~mm}$ wide tumour tile phantom. Since the width of the phantom is smaller than the sensitive detector dimension, the distribution shows a peak corresponding to the alignment centred over the sample.

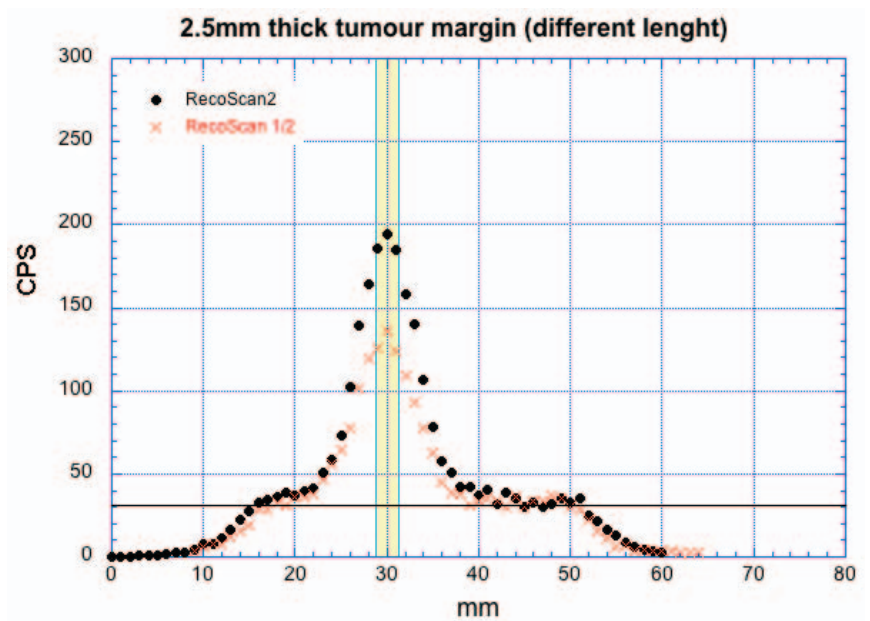

Fig. 15. Scan over a $2.5 \times 10 \mathrm{~mm}$ tumour margin. The red cross refers to the same scan performed on a smaller $2.5 \times 5 \mathrm{~mm}^{2}$ sample. 